\title{
Is There a Fingerprint Pattern in the Image?
}

\author{
Soweon Yoon and Anil K. Jain \\ Department of Computer Science and Engineering \\ Michigan State University, East Lansing, MI, U.S.A. \\ \{yoonsowo, jain\}@cse.msu.edu
}

\begin{abstract}
A fingerprint orientation field has distinct characteristics which can differentiate fingerprints from any other flow patterns: it has a specific number of singular points (cores and deltas), the configuration of singular points follows a certain spatial distribution, and its global shape is like an arch. In this paper, we propose a global fingerprint orientation field model, represented in terms of ordinary differential equations, which does not require any prior information such as singular points or orientation of a fingerprint. Further, the model requires only a small number of polynomial terms to represent the global fingerprint orientation field. The coefficients of the model are found subject to the constraints on the total number of singular points (i.e., 0 , 2, or 4) in a fingerprint. The proposed model is used to distinguish fingerprint images from non-fingerprint images and altered fingerprints by measuring the abnormality in the orientation field of the image.
\end{abstract}

\section{Introduction}

Fingerprint patterns can be characterized by (i) ridge frequency, (ii) orientation field, and (iii) minutiae distribution. Fingerprint ridges are spaced almost equidistantly; ridge period is typically around 10 pixels in 500 ppi fingerprint images. Fingerprint orientation field (often called ridge flow) is smooth and has a global shape of an arch, except in local regions around singular points (i.e., core and delta). Almost all fingerprints fall into one of the following three categories according to singularity: (i) no singularity (arch type), (ii) one core and one delta (left-loop, right-loop, and tentedarch type), and (iii) two cores and two deltas (whorl ${ }^{1}$ and twin-loop type). The configuration of cores and deltas in a fingerprint also follows a specific spatial distribution [11]. Minutiae distribution in a fingerprint has a tendency to be dense around singular points since the ridge flow converges or diverges around singular points while maintaining the

\footnotetext{
${ }^{1} \mathrm{~A}$ whorl can be viewed as consisting of two cores.
}

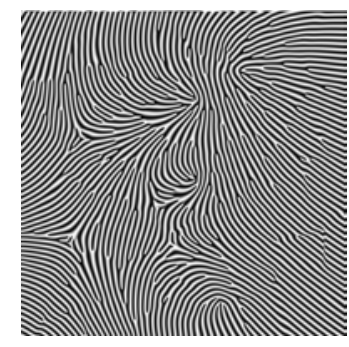

(a) NFIQ $=1, F=0.42$

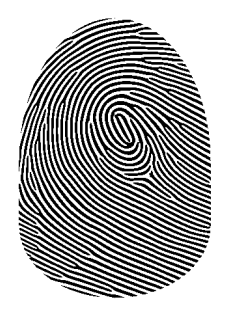

(c) NFIQ $=2, F=0.81$

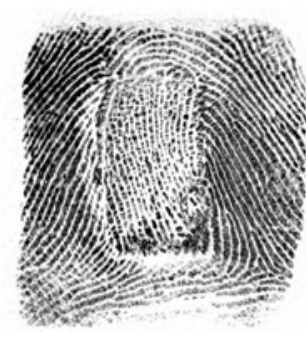

(b) NFIQ $=3, F=0.47$

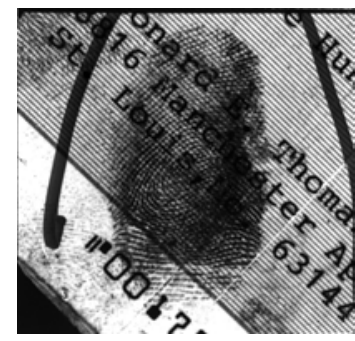

(d) $\mathrm{NFIQ}=2, F=0.72$
Figure 1. Images containing fingerprint-like pattern. (a) Synthesized image obtained by iterative contextual filtering [10], (b) altered fingerprint, where the central part of the fingerprint has been transplanted from a different friction ridge skin, (c) synthesized fingerprint by SFinGe [10], and (d) latent fingerprint from the NIST SD27 [1]. The NFIQ value [25] and the fingerprintness value $(F)$ of the orientation field are shown. The NFIQ gives one of the five discrete values from 1 (the highest quality) to 5 (the lowest quality). The fingerprintness value ranges from 0 (the lowest) to 1 (the highest).

ridge frequency [13].

To assess the quality of the fingerprint pattern, the features used to measure local properties (i.e., clarity of ridge structure) and global properties (e.g., continuity of orientation field or energy concentration in the frequency domain over the entire fingerprint region) have been utilized [6]. However, lack of global constraints that can specifically distinguish a valid fingerprint pattern from any other types of non-fingerprint images reveals the limitations of the exist- 
Table 1. Global fingerprint orientation field models. Note that $n$ is the order of the basis function or polynomial and $N_{s}$ is the number of singular points detected in the image. If the model requires singular points as input, the number of singular points is not included in the number of parameters to be estimated.

\begin{tabular}{lllll}
\hline Type & Model & \# Parameters & Singular Points & Fingerprint-specific Features \\
\hline Approximation & Piecewise linear functions [26] & $10 N_{s}$ & Estimated & Not utilized \\
methods & Polynomials [15] & $(n+1)(n+2)$ & Estimated & Not utilized \\
& Fourier series [28] & $2(2 n+1)^{2}$ & Estimated & Not utilized \\
& Legendre polynomials [22] & $(n+1)(n+2)$ & Estimated & Not utilized \\
\hline $\begin{array}{l}\text { Deterministic } \\
\text { mathematical } \\
\text { models }\end{array}$ & Rational polynomial function [23] & 1 & Required & Not utilized \\
& Constrained nonlinear & $(n+1)(n+2)-3 N_{s}$ & Estimated & Constraints on coefficients \\
& phase portrait model [20] & & & from singular points \\
& Quadratic differentials [18] & 5 & Required & Arch-shaped global field \\
& Peripheral models [27] & 2 for each model & Estimated & Arch-shaped global field \\
& Proposed model & 17 & Not required & Number of singular points \\
\hline
\end{tabular}

${ }^{\mathrm{a}}$ The number of coefficients here is only for the peripheral models, not including parameters in the Fourier expansion [28].

ing fingerprint quality measures to detect abnormality in the images shown in Fig. 1. Fig. 1a is a synthesized texture image using an iterative contextual filtering approach [10]; it has a valid fingerprint ridge frequency but its orientation field does not follow that of a fingerprint pattern. Fig. 1b is a fingerprint whose ridge structure in the central part of the finger has been transplanted from other friction ridge skin. Although the quality of local ridge structure in Fig. $1 \mathrm{~b}$ is good, the orientation field shows discontinuity at the boundaries of the altered region. Fig. 1c is a synthesized fingerprint image using SFinGe (Synthetic Fingerprint Generator) [10]; while the ridge frequency and the orientation field of this fingerprint image appear to be realistic, a large portion of the fingerprint area has ridges that flow parallel to each other without producing any minutiae. Fig. 1d is an example of a typical latent fingerprint which is found at crime scenes. Although the quality of the latent print in this image is fairly good, the background line structure with similar frequency value to a fingerprint ridge frequency makes it difficult to locate the fingerprint pattern in the image and extract features (e.g., orientation field and minutiae).

The most salient characteristic of a fingerprint pattern that distinguishes it from any other textured patterns is its orientation field. Global orientation field models for fingerprints have been developed primarily based on the following two approaches: (i) approximation methods, and (ii) deterministic mathematical models (see Table 1). Approximation methods represent the global orientation field with a set of piecewise linear functions [26], a set of polynomials [15], or a set of basis functions such as Fourier series [28] or Legendre polynomials [22]. A fingerprint orientation field can be represented as a vector consisting of the coefficients of the basis functions, and fingerprints can be clustered in feature space. However, since the coefficient vectors are not invariant to rotation and translation, the location and orientation of a fingerprint have to be determined first before es- timating the coefficients of the basis functions. In addition, when high order basis functions are used to represent fingerprints with high curvature (e.g., fingerprints with singular points), these models can fit well to virtually any flow pattern.

Deterministic mathematical models, on the other hand, incorporate unique characteristics of fingerprint orientation field such as local field around singular points [18, 20,23] and global field shaped like an arch $[18,27]$. Sherlock et al. [23] proposed a rational polynomial function which gives topological behavior of the global fingerprint ridge flow induced by singular points. Since the model in [23] requires only location and type of the singularities of a fingerprint, it does not reflect the actual ridge flow of the fingerprint. Also, in Sherlock et al.'s approach, there is no model for arch type of fingerprints which does not contain any singular points. Li et al. [20] proposed a constrained nonlinear phase portrait model which represents the $x$-derivative and $y$-derivative of the doubled orientation field with $n$th order polynomials and finds coefficients of the polynomials subject to the constraints derived from the local phase portrait behavior around singular points. Huckemann et al. [18] proposed a model based on quadratic differentials that describes local fields around core, delta, and whorl, and global field simulating arch-shaped field. The coordinates of the fingerprint (translation, rotation, scale, and aspect ratio of the axes) are estimated during model fit, while the singular points are required as input to determine the local orientation field. Wang et al. [27] proposed two peripheral models: a modified cosine function used in [9] and a fluid model which simulates the 2-dimensional flow of a uniform stream toward a cylinder.

Most deterministic mathematical models require information about singular points. The rational polynomial model [23] and the quadratic differential model [18] assume that actual location and type of the singular points of a fin- 
gerprint are given. The constrained nonlinear phase portrait model [20] estimates singular points by computing the Poincaré index [7] of the orientation field obtained from image gradient. However, the singular point information is not typically available as a prior knowledge in practice, but is extracted automatically from the image. Accurate detection of singular points is still a challenging problem especially in noisy fingerprints. If the information about singular points is not correct, the deterministic mathematical models give poor estimates of the orientation field.

In this paper, we propose a global orientation field model that incorporates a unique characteristic of fingerprint flow pattern, namely the number of singular points. First, the rational polynomial model [23] which provides a nice topology of fingerprint ridge flow with singular points is converted to a form of ordinary differential equations (ODE). Second, a generalized ODE model is proposed, which utilizes simpler polynomials and a smaller number of independent coefficients (i.e., 16 parameters in the model and 1 parameter for rotation) compared to the polynomial model in [15] and the constrained nonlinear phase portrait model in [20]. Third, the constraints on the number of singular points $(0,2$, or 4$)$ are applied to estimate the model parameters. Finally, the degree of similarity of a given image to a fingerprint pattern, called fingerprintness, is measured by computing the difference between the orientation field of the image and the orientation field fit by the proposed model. The fingerprintness is then used to distinguish fingerprint images from non-fingerprint images (e.g., natural scene images, other biometric modality images such as face and iris) and altered fingerprints.

\section{Global Fingerprint Orientation Field Model}

Orientation field of a fingerprint, $\theta(x, y)$, describes the tangential direction of the ridges at a point $(x, y)$, where $0 \leq \theta(x, y)<\pi$. Since the orientation has $\pi$-ambiguity (i.e., two different vectors, one directed at $\theta$ and the other directed at $(\theta+\pi)$, have the same orientation), the orientation field is often converted to a vector field by doubling the angles.

A 2-dimensional vector field can be represented by the first-order ODE as follows:

$$
\dot{x}=f(x, y) \quad \text { and } \quad \dot{y}=g(x, y)
$$

where $f(x, y)$ and $g(x, y)$ are real-valued functions. Given $f(x, y)$ and $g(x, y)$, the orientation field can be uniquely determined as:

$$
\theta(x, y)=\frac{1}{2} \tan ^{-1}\left(\frac{\dot{y}}{\dot{x}}\right)=\frac{1}{2} \tan ^{-1}\left(\frac{g(x, y)}{f(x, y)}\right) .
$$

The $x$-isocline in differential equations is the set of points where $\dot{x}=0$; that is, the $x$-isocline of the system in Eq. (1) is the set of points where $f(x, y)=0$ [17]. Similarly, the $y$-isocline of the system is the set of points where $\dot{y}=0$, i.e., $g(x, y)=0$. A singularity occurs at a point where both $\dot{x}=0$ and $\dot{y}=0$; that is, the $x$-isocline and the $y$-isocline intersect.

\subsection{From Rational Polynomial Function to ODE}

Sherlock et al. [23] proposed a fingerprint orientation field model with rational polynomial function:

$$
Q(z)=\frac{\left(z-z_{1}\right)\left(z-z_{2}\right) \cdots\left(z-z_{M}\right)}{\left(z-p_{1}\right)\left(z-p_{2}\right) \cdots\left(z-p_{N}\right)}
$$

where $z=x+i y,\left\{z_{m}\right\}_{1 \leq m \leq M}$ and $\left\{p_{n}\right\}_{1 \leq n \leq N}$ are cores and deltas, respectively, in the complex domain. Given this model, the orientation field from $Q(z)$ is obtained by:

$$
\theta(z)=\frac{1}{2} \arg \left(e^{i 2 \theta_{\infty}} \cdot Q(z)\right)
$$

where $\theta_{\infty}$ is the orientation of the fingerprint.

In Eq. (3), the set of points where the phase part is equal to $(2 k+1) \pi / 2, k$ is an integer, corresponds to $x$-isocline of its ODE representation. Similarly, the set of points where the phase part is equal to $k \pi, k$ is an integer, corresponds to $y$-isocline. For loop type of fingerprints (with one core and one delta), the $x$-isocline (Eq. (5)) and the $y$-isocline (Eq. (6)) are in a form of a circle and a line, respectively:

$$
\begin{gathered}
\left(x-x_{o}\right)^{2}+\left(y-y_{o}\right)^{2}-r_{o}^{2}=0, \\
a x+b y+c=0 .
\end{gathered}
$$

Fig. 2 illustrates the orientation field of the loop model from the rational polynomial function and its vector field with $x$-isocline and $y$-isocline.

Similarly, the $x$-isocline (Eq. (7)) and the $y$-isocline (Eq. (8)) for double-loop type of fingerprints (with two cores and two deltas) in the rational polynomial model can be represented in the following forms:

$$
\begin{aligned}
& \left(x^{2}+y^{2}\right)\left\{\left(x-x_{o}\right)^{2}+\left(y-y_{o}\right)^{2}-r_{o}^{2}\right\} \\
+ & A x^{2}+B x y-A y^{2}+C x+D y+E=0, \\
& \left(x^{2}+y^{2}\right)(a x+b y+c) \\
+ & A x^{2}+B x y-A y^{2}+C x+D y+E=0 .
\end{aligned}
$$

The $x$-isocline and $y$-isocline for double-loop fingerprints also consist of geometric forms: line, circle, and hyperbola. Fig. 3 shows the double-loop model from the rational polynomial function with $x$-isocline and $y$-isocline in the vector field. Note that the parameters in Eqs. (5) - (8) are solely determined by the given singular points. 


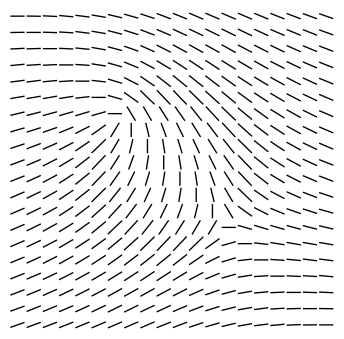

(a)

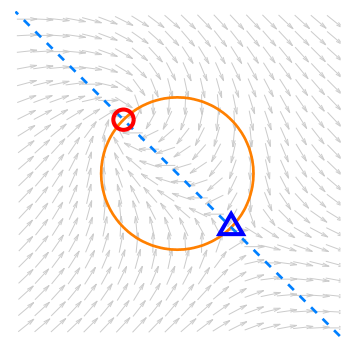

(b)

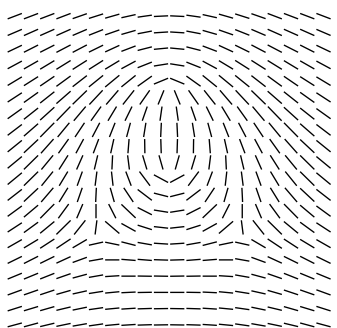

(a)

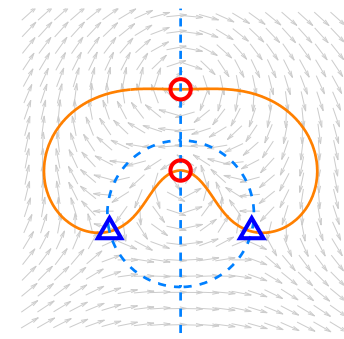

(b)
Figure 2. Model for a loop type of fingerprint based on the rational polynomial function. (a) Orientation field and (b) vector field with $x$-isocline (orange solid curve) and $y$-isocline (blue dotted line) with singular points (a core is marked as a circle and a delta is marked as a triangle).

\subsection{Generalized Orientation Field Model in ODE}

Based on the rational polynomial function converted into the ODE form, a generalized fingerprint orientation field model in nonlinear ODE is derived from the double-loop model which is the most complicated case. The generalized model is shown in Eq. (9): $f(x, y)$ is the 4th order polynomial with fewer polynomial terms and the coefficients of the 3rd order polynomial terms are correlated; $g(x, y)$ is the 3 rd order polynomial with full polynomial terms, but the coefficients of the 3 rd order polynomial terms are correlated. Thus, there are 8 coefficients for each $f(x, y)$ and $g(x, y)$.

$$
\begin{aligned}
\dot{x}=f(x, y) & =x^{4}+2 x^{2} y^{2}+y^{4}+a x^{3}+b x^{2} y+a x y^{2}+b y^{3} \\
& +A x^{2}+B x y+C y^{2}+D x+E y+F, \\
\dot{y}=g(x, y) & =a^{\prime} x^{3}+b^{\prime} x^{2} y+a^{\prime} x y^{2}+b^{\prime} y^{3} \\
& +A^{\prime} x^{2}+B^{\prime} x y+C^{\prime} y^{2}+D^{\prime} x+E^{\prime} y+F^{\prime} .
\end{aligned}
$$

\subsubsection{Constraints on Singularity}

As a global constraint which characterizes a fingerprint pattern, the number of singular points in the modeled orientation field needs to be restricted to 0,2 , or 4 . Considering that the singular points occur when $x$-isocline and $y$-isocline intersect, we want to find a constraint on the number of real roots of the following polynomial system:

$$
f(x, y)=0 \quad \text { and } \quad g(x, y)=0 .
$$

According to Hermite's theorem, the constraint which specifies the number of real roots of a polynomial system can be found as follows [21]:

1. Find the Gröbner bases [5] of the polynomial system, $G$.

2. Construct a set of monomials that are not multiples of the leading terms of the polynomials in $G, M=$ $\left\{m_{1}, m_{2}, \ldots, m_{P}\right\}$.
Figure 3. Model for a double-loop type of fingerprint based on the rational polynomial function. (a) Orientation field and (b) vector field with $x$-isocline (orange solid curve) and $y$-isocline (blue dotted curve) with singular points (cores are marked as circles and deltas are marked as triangles).

3. Compute normal forms ${ }^{2}$ of each monomial in $M$ multiplied by $x$ and $y$; that is, for each monomial $m_{p}$ in $M$, the normal forms of $x m_{p}$ and $y m_{p}$ are computed.

4. Construct coefficient matrices, $\mathbf{P}_{x}$ and $\mathbf{P}_{y}$, where the $(i, j)$-th entry of $\mathbf{P}_{x}$ is the coefficient of the term $m_{i}$ in the normal form of $x m_{j}$ (similarly, the $(i, j)$-th entry of $\mathbf{P}_{y}$ is the coefficient of the term $m_{i}$ in the normal form of $y m_{j}$ ).

5. Construct a trace matrix $\mathbf{T}_{1}$ which is defined as follows:

$$
\mathbf{T}_{1}=\left[\begin{array}{ccc}
\operatorname{Tr}\left(m_{1} m_{1}\right) & \cdots & \operatorname{Tr}\left(m_{1} m_{P}\right) \\
\vdots & & \vdots \\
\operatorname{Tr}\left(m_{P} m_{1}\right) & \cdots & \operatorname{Tr}\left(m_{P} m_{P}\right)
\end{array}\right]
$$

where $\operatorname{Tr}\left(m_{i} m_{j}\right)$ is the trace of a matrix $\mathbf{P}_{x}^{k_{i}+k_{j}} \mathbf{P}_{y}^{l_{i}+l_{j}}$ when $m_{i}=x^{k_{i}} y^{l_{i}}$ and $m_{j}=x^{k_{j}} y^{l_{j}}$.

6. Hermite's Theorem The signature of $\mathbf{T}_{1}$ is equal to the number of real roots of the polynomial system; the signature of a real symmetric matrix is the difference between the numbers of positive and negative eigenvalues.

Note that eigenvalues of $\mathbf{P}_{x}$ and $\mathbf{P}_{y}$ are used to find singular points of a fingerprint (i.e., real roots of the polynomial system).

\subsubsection{Parameter Estimation}

A foreground mask, $\mathcal{R}$, consists of the local blocks with a sufficient dynamic range in image intensity. The dynamic range of a local block, $D(x, y)$, is defined as the difference between the minimum and the maximum grayscale intensity after discarding $10 \%$ of the highest and the lowest grayscale intensities in the block [29]. If $D(x, y)$ is greater than 20,

\footnotetext{
${ }^{2}$ Given the Gröbner bases $G$ of a polynomial system, the normal form of any polynomial is a polynomial which does not contain any term that is divisible by the leading terms of the polynomials in $G$ after a finite number of reductions.
} 


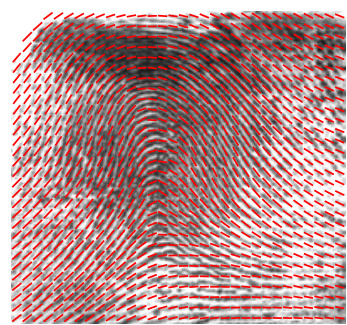

(a)

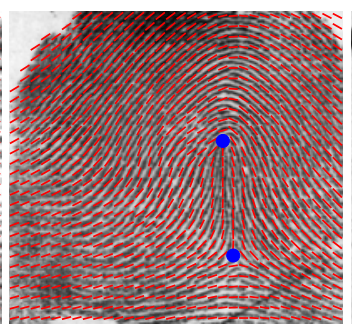

(b)

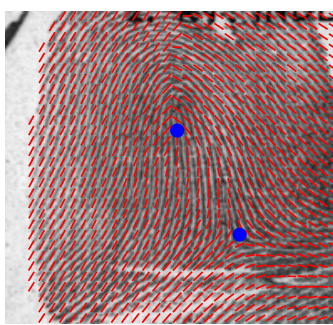

(c)

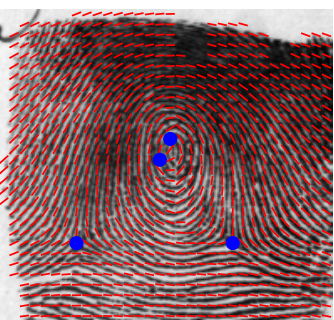

(d)

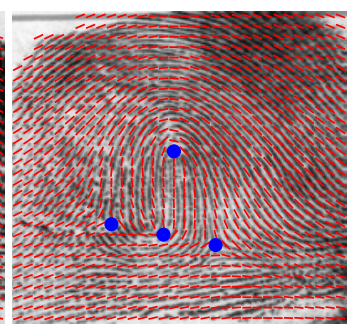

(e)

Figure 4. Orientation field and singular points estimated by the proposed model for (a) arch, (b) tented arch, (c) loop, (d) whorl, and (e) twin-loop type fingerprint.

the block is determined as foreground. The orientation field in the foreground mask is computed by image gradient [7]. To ensure that the modeled orientation field well reflects the high curvature ridge structure including singular region, the absolute value of the determinant of a characteristic matrix in vector field linearization ${ }^{3}$ [24] is used as a weight matrix in parameter estimation.

The objective function for parameter estimation is:

$$
\min \sum_{(x, y) \in \mathcal{R}} \sin ^{2}[\theta(x, y)-\hat{\theta}(x, y)] \cdot[\omega+(1-\omega) c(x, y)]
$$$$
\text { subject to the signature of } \mathbf{T}_{1} \text { as } 0,2 \text {, or } 4 \text {, }
$$

where $\theta(x, y)$ is the orientation field extracted from the image, $\hat{\theta}(x, y)$ is the modeled orientation field, $\omega$ is a weight ( $\omega=0.2$ is used in the paper), $c(x, y)$ is the map of the absolute value of the determinant of a characteristic matrix at $(x, y)$, and $\mathbf{T}_{1}$ is the trace matrix in Eq. (10).

While the origin of the image coordinate system is set to the center of the image (translation parameters are not needed), the rotation parameter needs to be estimated. The modeled orientation field with rotation angle $r$ is obtained as follows:

$$
\hat{\theta}(\mathbf{x})=\frac{1}{2} \tan ^{-1}\left(\frac{\mathbf{R}^{2} g\left(\mathbf{R}^{-1} \mathbf{x}\right)}{\mathbf{R}^{2} f\left(\mathbf{R}^{-1} \mathbf{x}\right)}\right),
$$

where $\mathbf{x}=\left[\begin{array}{l}x \\ y\end{array}\right]$ and $\mathbf{R}=\left[\begin{array}{rr}\cos r & -\sin r \\ \sin r & \cos r\end{array}\right]$.

In total, 17 parameters, including a rotation parameter, are estimated during model fit. The constrained nonlinear optimization problem is solved by interior-point algorithm [8]. For the initial parameter estimation, the objective function in Eq. (11) is used without applying the constraints. Then the parameters are estimated by applying one of the three constraints for the number of singular points $(0$, 2 , and 4) at each time. Only the estimated parameters which do not violate the constraint are considered as valid. Fig. 4 shows the orientation field and singular points estimated by the proposed model for various types of fingerprints.

\footnotetext{
${ }^{3}$ Note that the determinant of a characteristic matrix of the linearized vector field is non-zero at singular points.
}

\section{Defining a Fingerprint Pattern}

One of the fundamental questions in image analysis is to determine if a given image contains a specific type of objects or textures [19]. In fingerprint identification, it is important to ensure that a given image contains valid fingerprint pattern which is appropriate for fingerprint matching. A global orientation field model can be used (i) to check the validity of the input image to Automated Fingerprint Identification Systems (AFIS), and (ii) to check the integrity of exemplars (rolled or slap fingerprints) in a reference fingerprint database. If a valid fingerprint image of a good quality is given as input to an AFIS, a global orientation field model specifically designed to represent fingerprint patterns is expected to fit well to the orientation field of the input image. If the input image is a poor quality fingerprint which does not provide reliable orientation field as input to the model or the input is not a fingerprint image, the orientation field obtained from the model is expected to deviate significantly from the orientation field of the image. Fig. 5a shows an example of a good quality fingerprint image and its orientation field difference map (difference of image orientation field and modeled orientation field); Fig. 5b shows an example of a non-fingerprint image and its orientation field difference map.

As a measure of the similarity of a given image to a valid fingerprint pattern, we introduce a term, fingerprintness $(F)$, which indicates the similarity of a given image to fingerprint patterns and is a normalized score ranged from 0 to 1 . In this paper, we measure fingerprintness in terms of the orientation field of an image. A 10-dimensional feature vector is constructed by concatenating 5-dimensional feature vectors (contrast, energy, and homogeneity of the gray-level co-occurrence matrix (GLCM) [16], and the mean and standard deviation) from (i) the orientation field difference map in the foreground region $\mathcal{R}$, and (ii) the orientation field difference map multiplied by the grayscale dynamic range map $D$. A support vector machine (SVM) classifier is trained by a set of fingerprint images and a set of non-fingerprint images, and the $F$ value for a test image is determined as the 


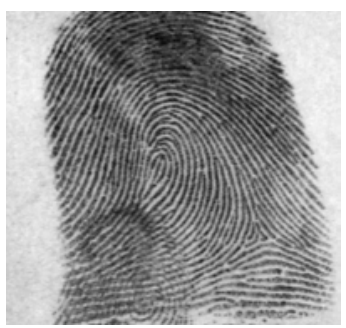

(a)

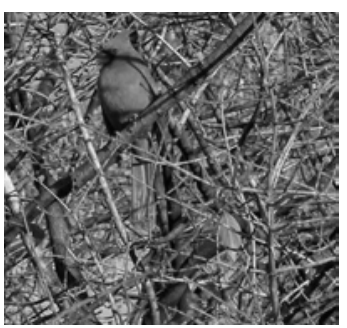

(e)

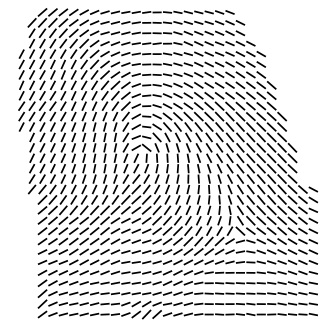

(b)

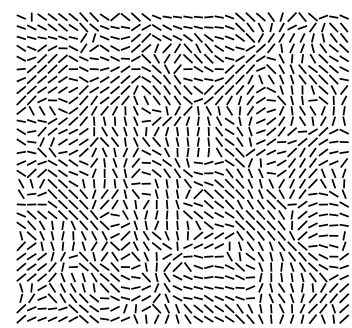

(f)

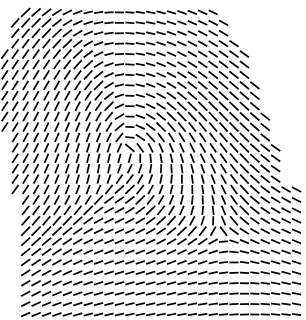

(c)

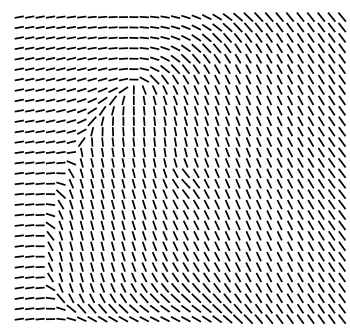

(g)

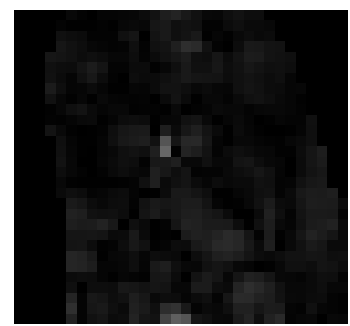

(d)

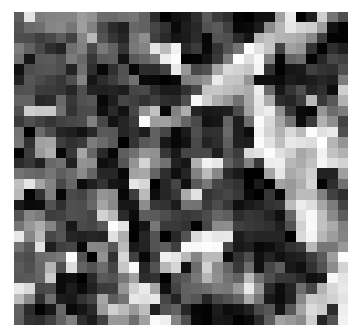

(h)

Figure 5. Orientation field difference map. (a)-(d) A fingerprint image, its orientation field, the orientation field from the model, and the orientation field difference map; (e)-(h) a non-fingerprint image, its orientation field, the orientation field from the model, and the orientation field difference map.

normalized decision value from the SVM classifier.

Prior to obtaining the $F$ value of an image, the image can be rejected by the size of $\mathcal{R}$ and the validity of the model fit. If the foreground region $\mathcal{R}$ takes less than $30 \%$ of the entire image area, the image is considered to consist of blocks with smooth variation in grayscale intensity which is not a desirable property of a good quality fingerprint. Also, if the model does not find any feasible solution satisfying any of the three constraints on the number of singularities, the input image is also rejected.

\section{Experimental Results}

The proposed fingerprint orientation field model is used to measure the abnormality in the orientation field of a given image compared to a fingerprint pattern. Since we are interested in abnormality detection, positive class refers to non-fingerprint or altered fingerprint class, and negative class refers to fingerprint class. We collect fingerprint and non-fingerprint images from the following sources: (i) first 2,000 fingerprint impressions in the NIST SD4 [2], and (ii) 2,000 images drawn from the ImageNet dataset [14] (100 object classes are randomly selected with 20 images per class), 2,000 face images from the FERET database [3], 2,000 iris images from the MBGC database [4], and 2,000 images from the altered fingerprint database [30].

Table 2 summarizes the number of images that are detected as non-fingerprints by (i) size of $\mathcal{R}$, (ii) validity of model fit, and (iii) abnormality in orientation field as a fingerprint pattern.

A 2-class classification problem is solved by using the 10-dimensional feature vector extracted from the orientation field difference map to discriminate non-fingerprint images (ImageNet images, faces, and irises) that are passed through the rejection criteria from fingerprint images. An SVM with radial basis function [12] is used as a classifier and the performance is evaluated by 10 -fold cross validation. Fig. 6 shows the Receiver Operating Characteristic (ROC) curve for the average performance over 10 folds.

The proposed model is also used to distinguish altered fingerprints from normal fingerprint images. Fig. 7 shows the ROC curves in detecting altered fingerprints with (i) the proposed model with GLCM features extracted from the orientation field difference map, and (ii) the polynomial model with histogram features from the orientation field difference map (without multiplication with $D$ ) used in [30]. The orientation field model and salient feature extraction help improve the altered fingerprint detection accuracy significantly.

Figs. 8 and 9 show examples of fingerprint images with low fingerprintness values. If the model fit is wrong (Fig. $8 \mathrm{a}$ is a double-loop type of fingerprint, but the model fits a loop type), a high response in the orientation field difference map is observed. Fig. 8b shows a case where the fingerprint is potentially altered which explains its low fingerprintness value. On the other hand, if the orientation field extracted from the image is noisy due to the poor quality of fingerprint ridges (e.g., Fig. 9), the orientation field difference can also be high although the proposed model leads to a good estimation of the orientation field.

Fig. 10 shows an example of non-fingerprint image 
Table 2. Detection results for non-fingerprint images and altered fingerprints.

\begin{tabular}{l|c|cccc}
\hline Detection Criteria & NIST SD4 & ImageNet & Face & Iris & Altered Fingerprints \\
\hline Size of $\mathcal{R}$ & 1 & 326 & 1,862 & 1,574 & 43 \\
Validity of model fit & 6 & 28 & 3 & 13 & 21 \\
$F<F_{o}{ }^{\text {a }}$ at FPR & 20 & 1,477 & 119 & 367 & 1,713 \\
\hline Total number of detected images & 27 & 1,831 & 1,984 & 1,954 & 1,777 \\
(Detection rate $(\%))$ & $(1.35 \%)$ & $(91.55 \%)$ & $(99.20 \%)$ & $(97.70 \%)$ & $(88.85 \%)$ \\
\hline
\end{tabular}

${ }^{\mathrm{b}}$ The thresholds $F_{o}$ corresponding to false positive rate (FPR) of $1 \%$ are 0.62 and 0.65 for non-fingerprint image detection and altered fingerprint detection, respectively.

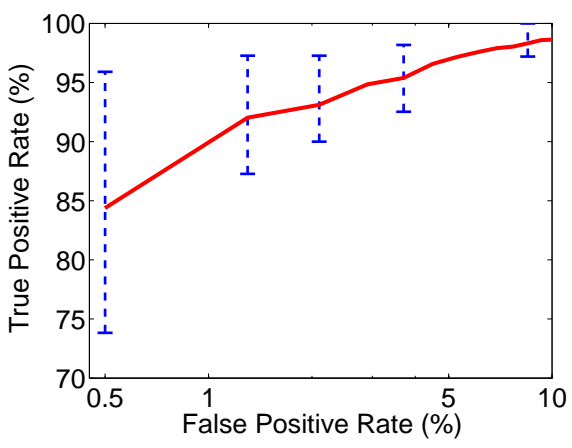

Figure 6. ROC curve for fingerprint vs. non-fingerprint (i.e., natural scenes, objects, faces, and irises) classification. The average performance (red line) and the minimum and maximum performances (blue bars) in the 10-fold cross validation are shown.

with high fingerprintness value where the image contains fingerprint-like orientation. Fig. 11 is a fingerprint with small altered region, which gives high fingerprintness value.

\section{Conclusions and Future Work}

Global fingerprint orientation field models are necessary to capture unique characteristics of a fingerprint pattern. Such models can be used to (i) distinguish fingerprints from any other types of texture pattern, (ii) represent the fingerprint orientation field in a compact form, and (iii) estimate the orientation field from noisy or partially missing ridge structures in fingerprints. The global orientation field models published in the literature are highly dependent on singular point information; if incorrect information about singularities is provided, the models are likely to fail to fit to the image. In this paper, we propose an ODE representation of the global orientation field which is derived from the rational polynomial model, but does not require singular point information as input. A set of constraints on the number of singular points in fingerprints is applied to find coefficients of the model. The proposed model is used to detect abnormality in the orientation field of a given image and then classify it as a fingerprint or a non-fingerprint pattern.

Future work on global orientation field modeling and fingerprintness measure includes:

1. Improving the global orientation field model by incorporating other fingerprint-specific features such as config-

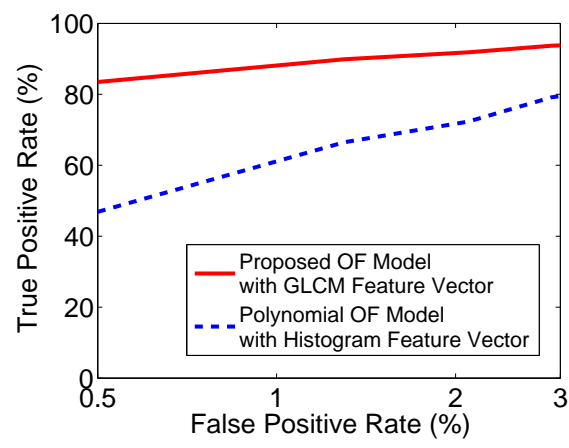

Figure 7. ROC curve for fingerprint vs. altered fingerprint classification. The proposed orientation field model with GLCM features is compared to the polynomial model with histogram features used in [30].

uration of singular points and arch-shaped global field;

2. Including features from ridge structure and minutiae distribution into the definition of fingerprintness.

\section{References}

[1] NIST Special Database 27: Fingerprint Minutiae from Latent and Matching Tenprint Images.

[2] NIST Special Database 4: NIST 8-Bit Gray Scale Images of Fingerprint Image Groups (FIGS).

[3] The Facial Recognition Technology (FERET) Database.

[4] Multiple Biometric Grand Challenge (MBGC) Database.

[5] W. W. Adams and P. Loustaunau. An Introduction to Gröbner Bases (Graduate Studies in Mathematics, Vol 3). American Mathematical Society, 1994.

[6] F. Alonso-Fernandez, J. Fierrez, J. Ortega-Garcia, J. Gonzalez-Rodriguez, H. Fronthaler, K. Kollreider, and J. Bigun. A Comparative Study of Fingerprint Image-Quality Estimation Methods. IEEE Trans. IFS, 2(4):734-743, 2007.

[7] A. M. Bazen and S. H. Gerez. Systematic Methods for the Computation of the Directional Fields and Singular Points of Fingerprints. IEEE Trans. PAMI, 24(7):905-919, 2002.

[8] R. H. Byrd, M. E. Hribar, and J. Nocedal. An Interior Point Algorithm for Large-Scale Nonlinear Programming. SIAM J. Optimization, 9(4):877-900, 1999.

[9] R. Cappelli, A. Lumini, D. Maio, and D. Maltoni. Fingerprint Image Reconstruction from Standard Templates. IEEE Trans. PAMI, 29(9):1489-1503, 2007.

[10] R. Cappelli, D. Maio, and D. Maltoni. Synthetic FingerprintDatabase Generation. In Proc. ICPR, pages 744-747, 2002. 
[11] R. Cappelli and D. Maltoni. On the Spatial Distribution of Fingerprint Singularities. IEEE Trans. PAMI, 31(4):742-748, 2009.

[12] C.-C. Chang and C.-J. Lin. LIBSVM: a library for support vector machines, 2001.

[13] Y. Chen and A. K. Jain. Beyond Minutiae: A Fingerprint Individuality Model with Pattern, Ridge and Pore Features. In Proc. ICB, pages 523-533, 2009.

[14] J. Deng, W. Dong, R. Socher, L.-J. Li, K. Li, and L. Fei-fei. Imagenet: A Large-Scale Hierarchical Image Database. In Proc. CVPR, pages 248-255, 2009.

[15] J. Gu, J. Zhou, and D. Zhang. A Combination Model For Orientation Field of Fingerprints. Pattern Recognition, 37(3):543-553, 2004.

[16] R. M. Haralick, K. Shanmugam, and I. Dinstein. Textural Features for Image Classification. IEEE Trans. SMC, SMC3(6):610-621, 1973.

[17] M. W. Hirsch, S. Smale, and R. L. Devaney. Differential Equations, Dynamical Systems, and an Introduction to Chaos (Second Edition). Elsevier Academic Press, 2004.

[18] S. Huckemann, T. Hotz, and A. Munk. Global Models for the Orientation Field of Fingerprints: An Approach Based on Quadratic Differentials. IEEE Trans. PAMI, 30(9):15071519, 2008.

[19] K. Karu, A. K. Jain, and R. M. Bolle. Is There Any Texture in the Image? Pattern Recognition, 29(9):1437-1446, 1996.

[20] J. Li, W.-Y. Yau, and H. Wang. Constrained Nonlinear Models of Fingerprint Orientations with Prediction. Pattern Recognition, 39(1):102-114, 2006.

[21] S. Petitjean. Algebraic Geometry and Computer Vision: Polynomial Systems, Real and Complex Roots. J. Mathematical Imaging and Vision, 10:191-220, 1999.

[22] S. Ram, H. Bischof, and J. Birchbauer. Modelling Fingerprint Ridge Orientation Using Legendre Polynomials. Pattern Recognition, 43(1):342-357, 2010.

[23] B. G. Sherlock and D. M. Monro. A Model for Interpreting Fingerprint Topology. Pattern Recognition, 26(7):10471055, 1993.

[24] C.-F. Shu and R. C. Jain. Vector Field Analysis for Oriented Patterns. IEEE Trans. PAMI, 16(9):946-950, 1994.

[25] E. Tabassi, C. Wilson, and C. Watson. Fingerprint Image Quality. NISTIR 7151, August 2004.

[26] P. R. Vizcaya and L. A. Gerhardt. A Nonlinear Orientation Model for Global Description of Fingerprints. Pattern Recognition, 29(7):1221-1231, 1996.

[27] Y. Wang and J. Hu. Global Ridge Orientation Modeling for Partial Fingerprint Identification. IEEE Trans. PAMI, 33(1):72-87, 2010.

[28] Y. Wang, J. K. Hu, and D. Phillips. A Fingerprint Orientation Model Based on 2D Fourier Expansion (FOMFE) and Its Application to Singular-Point Detection and Fingerprint Indexing. IEEE Trans. PAMI, 29(4):573-585, 2007.

[29] C. Watson, M. Garris, E. Tabassi, C. Wilson, R. M. McCabe, S. Janet, and K. Ko. NIST Biometric Image Software. www . nist.gov/itl/iad/ig/nbis.cfm.

[30] S. Yoon, J. Feng, and A. K. Jain. Altered fingerprints: Analysis and detection. IEEE Trans. PAMI, 34(3):451-464, 2012.

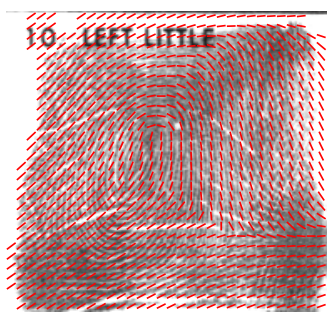

(a) $\mathrm{F}=0.55$

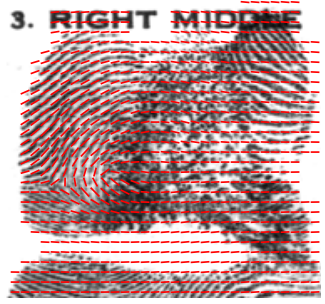

(b) $\mathrm{F}=0.62$
Figure 8. Fingerprint images with low fingerprintness values. (a) Failure in correct model fit and (b) potentially altered fingerprint. The modeled orientation fields are overlaid on the images.

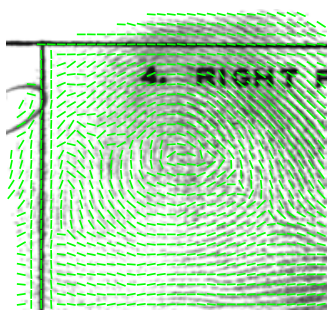

(a)

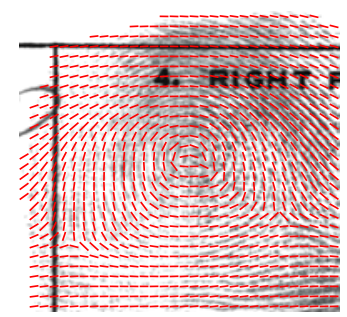

(b)
Figure 9. A noisy fingerprint image with low fingerprintness value $(\mathrm{F}=0.55)$. (a) Orientation field extracted from the image and (b) orientation field estimated by the proposed model.

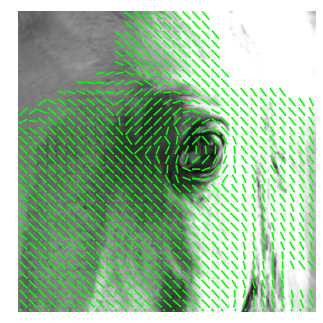

(a)

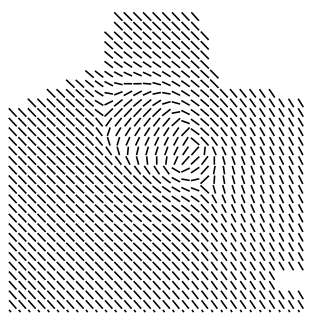

(b)
Figure 10. A non-fingerprint image with high fingerprintness value $(\mathrm{F}=0.87)$ due to fingerprint-like orientation field extracted from the image. (a) Orientation field extracted from the image and (b) modeled orientation field.

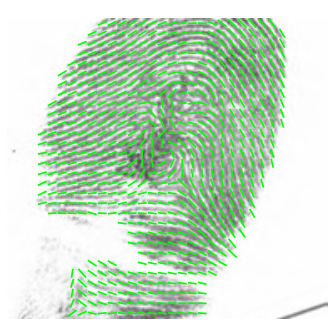

(a)

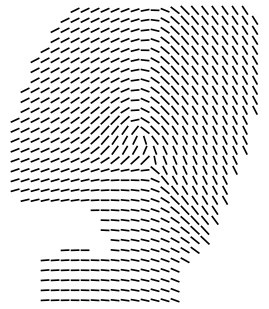

(b)
Figure 11. An altered fingerprint with high fingerprintness value $(\mathrm{F}=0.88)$ due to small altered region in (a). 